Check for updates

Cite this: RSC Adv., 2018, 8, 1055

Received 6th November 2017

Accepted 13th December 2017

DOI: $10.1039 / c 7 r a 12173 c$

rsc.li/rsc-advances

\title{
Biological activity and interaction mechanism of the diketopiperazine derivatives as tubulin polymerization inhibitors $\uparrow$
}

\author{
Zhenhua Tian, (D) a Yanyan Chu, ${ }^{\text {ac }}$ Hui Wang, ${ }^{a}$ Lili Zhong, ${ }^{a}$ Mengyan Deng ${ }^{a}$ \\ and Wenbao Li*abc
}

\begin{abstract}
Microtubules are a favorable target for development of anticancer agents. In this study, the anti-proliferative activities of plinabulin and six diketopiperazine derivatives were evaluated against human lung cancer cell line $\mathrm{NCl}-\mathrm{H} 460$ and human pancreatic cancer cell line BxPC-3. The inhibition activities on these microtubules were assessed by tubulin polymerization and immunofluorescence assays. To gain insight into the interaction mechanism of the derivatives and tubulin, a molecular dynamics simulation was performed. We discovered that the diketopiperazine derivatives could prevent tubulin assembly through conformational changes. Molecular Mechanics/Poisson-Boltzmann Surface Area (MM-PBSA) calculations showed that the trend of the binding free energies of these inhibitors was in agreement with the trend of their biological activities. Introducing hydrophobic groups into the A-ring was favorable for binding. Energy decomposition indicated that van der Waals interaction played an essential role in the binding affinity of tubulin polymerization inhibitors. In addition, the key residues responsible for inhibitor binding were identified. In summary, this study provided valuable information for development of novel tubulin polymerization inhibitors as anticancer agents.
\end{abstract}

\section{Introduction}

Microtubules (MTs) are typically formed by 13 protofilaments associating laterally in parallel to form a hollow and polar cylinder. Each protofilament consists of $\alpha, \beta$-tubulin heterodimers assembled with a head-to-tail dynamic configuration..$^{1-3}$ Polymerization dynamics are closely linked to the complex at MT plus end, which switches between phases of growth and disassembly. This so-called "dynamic instability" is controlled by the hydrolysis of GTP in $\beta$-tubulin upon polymerization. ${ }^{4}$ As a key component of cytoskeleton, MTs play diverse roles in cell division, cell migration and intracellular transport. ${ }^{5}$ They are considered as potential antitumor targets because destabilizing and stabilizing of MTs can perturb cell division. Currently, as shown in Fig. 1, four binding sites of microtubules have been identified: taxol, ${ }^{6}$ vinblastine, ${ }^{7}$ colchicine ${ }^{8}$ and laulimalide. ${ }^{9}$ Till date, the U.S. Food and Drug Administration (FDA) has approved several anti-tubulin agents targeting the taxol and vinblastine sites in cancer chemotherapy. ${ }^{\mathbf{1 0}, 11}$ However, the use

\footnotetext{
${ }^{a}$ School of Medicine and Pharmacy, Ocean University of China, Qingdao 266003, China.E-mail:wbli92128@163.com

${ }^{b}$ Innovation Center for Marine Drug Screening and Evaluation, Qingdao National Laboratory for Marine Science and Technology, Qingdao 266071, China ${ }^{c}$ Marine Biomedical Research Institute of Qingdao, Qingdao 266071, China

$\dagger$ Electronic supplementary information (ESI) available. See DOI: $10.1039 / \mathrm{c} 7 \mathrm{ra12173 \textrm {c }}$
}

of colchicine in cancer chemotherapy is restrained by its low therapeutic index. ${ }^{12,13}$ To overcome the clinical limitations, multiple efforts have been explored to develop the colchicine binding site inhibitors (CBSIs). However, these developed drug candidates failed due to undesirable toxicity and multidrug resistance (MDR). Therefore, it is essential to develop novel CBSIs with low toxicity and high efficacy.

Plinabulin was developed from the natural cyclic diketopiperazine (DKP) derivative "phenylahistin", which was isolated from Aspergillus ustus. ${ }^{\mathbf{1 4 1 5}}$ Currently, plinabulin is in phase III clinical trial for treatment of non-small cell lung cancer. ${ }^{16}$ For structure-activity relationship study, a series of diketopiperazine (DKP) derivatives were synthesized and their biological activities were evaluated. ${ }^{17,18}$ Notably, there was a correlation between the dissociation constants of the inhibitors binding to tubulin $\left(K_{\mathrm{d}}\right)$ and the $\mathrm{IC}_{50}$ values against human colon cancer cell lines HT-29.17,18

For further rational drug design, seven DKP derivatives including plinabulin were synthesized (Fig. 2), and their antiproliferative activities were evaluated against human pancreatic cancer cell line BxPC-3 and human lung cancer cell line NCI-H460. The structure-activity relationship and interaction mechanisms of the derivatives were explored by molecular docking combined with MD simulation. Both structural analysis and binding free energy calculations provided us valuable information for development of novel tubulin polymerization inhibitors as anticancer agents. 


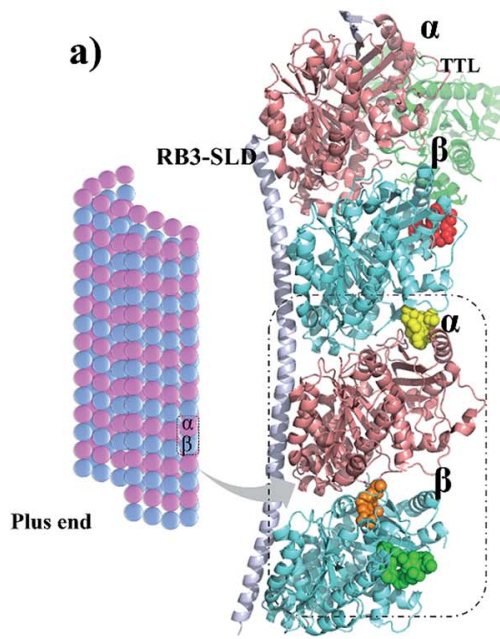

b)
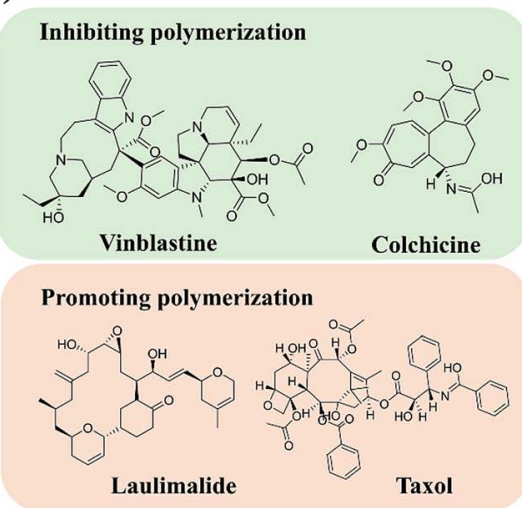

Fig. 1 (a) Four microtubule binding sites: colchicine (orange), laulimalide (red), taxol (green) and vinblastine (yellow). $\alpha$ and $\beta$ tubulins are shown in pink and blue, respectively. (b) Structures of the representative compounds.

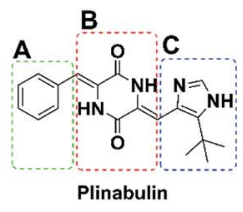

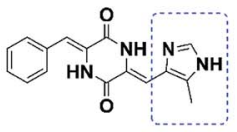

Compound a

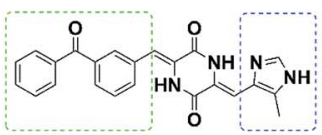

Compound d

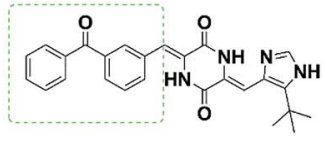

Compound $b$

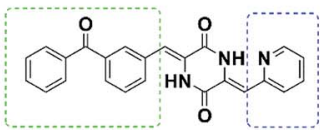

Compound e

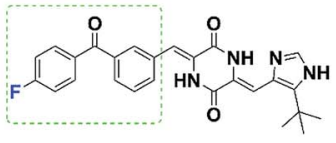

Compound c

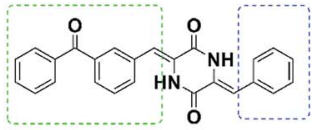

Compound $f$

Fig. 2 Structures of the synthesized diketopiperazine derivatives.

\section{Experimental}

\subsection{Synthesis of the diketopiperazine derivatives}

These derivatives were synthesized according to our previous published reaction routes with necessary modification. ${ }^{19}$ The structures were characterized by MS and ${ }^{1} \mathrm{H}$-NMR (see ESI $\dagger$ ). The purities of these compounds were determined by HPLC.

\subsection{SRB assay}

Human cancer cell lines NCI-H460 and BxPC-3 were purchased from American Type Cell Culture Collection (ATCC, USA). Sulforhodamine $\mathrm{B}$ (SRB) assay was widely used for in vitro measurement. Both human lung cancer cell line NCI-H460 and human pancreatic cancer cell line BxPC-3 were cultured in RPMI-1640 media with $2 \mathrm{mM}$ glutamine and $100 \mathrm{U}$ per $\mathrm{mL}$ penicillin. Next, BxPC-3 and NCI-H460 cells at logarithmic growth phase were seeded in 96-well plates at 5000 cells per well. Plinabulin derivatives at different concentrations were added into the wells. After incubation for $72 \mathrm{~h}, 50 \mu \mathrm{L}$ of stock solution of $50 \%$ TCA was added to each well at $4{ }^{\circ} \mathrm{C}$. Then, the 96-well plate was placed at $4{ }^{\circ} \mathrm{C}$ for $1 \mathrm{~h}$. Then, $70 \mu \mathrm{L}$ of $0.4 \%$ SRB $(\mathrm{w} / \mathrm{v})$ solution in $1 \%$ acetic acid was added to each well. The plate was placed at room temperature for $30 \mathrm{~min}$, and then washed with $1 \%$ acetic acid five times to remove unbound SRB. Then, $150 \mu \mathrm{L}$ of $10 \mathrm{mM}$ Trizma base solution was added into each well to solubilize the bound SRB. Finally, the absorbance of 96-well plate was measured using a microplate reader (SpectraMax I3, Molecular Devices, USA) at $540 \mathrm{~nm}$.

\subsection{In vitro tubulin polymerization assay}

Tubulin polymerization assay was performed according to the method described by Bonne et al. with appropriate 
modification..$^{\mathbf{2 0 2 1}}$ A commercial kit (BK011P) was purchased from Cytoskeleton (Danvers, MA, USA). The final tubulin solution contained $80.0 \mathrm{mM}$ piperazine- $N, N^{\prime}$-bis (2-ethanesulfonic acid) sequisodium salt ( $\mathrm{pH}$ 6.9), $2.0 \mathrm{mM} \mathrm{MgCl}_{2}, 0.5 \mathrm{mM}$ EGTA, $1 \mathrm{mM}$ GTP, and 15\% glycerol. The 96-well plate was warmed at $37{ }^{\circ} \mathrm{C}$ for $1 \mathrm{~min}$ after the compounds $(5 \mu \mathrm{L})$ were added. Plinabulin was used as a positive control. Then, $45 \mu \mathrm{L}$ of the tubulin solution was added into each well rapidly, and the plate was read immediately. Fluorescence was monitored (excitation at $360 \mathrm{~nm}$ and emission at $450 \mathrm{~nm}$ ) every $1 \mathrm{~min}$ for $20 \mathrm{~min}$ by a microplate reader (SpectraMax I3, Molecular Devices, USA).

\subsection{Immunofluorescence assay}

BxPC-3 cells were seeded onto poly-lysine treated coverslips in complete media and treated with $5 \mathrm{nM}$ compound for $24 \mathrm{~h}$. Then, the coverslips were washed three times with PBS. The fixed cells were incubated with $4 \%$ paraformaldehyde in PBS at room temperature for $15 \mathrm{~min}$. After rinsing three times with PBS in 5 min and blocking samples in 1\% bovine serum albumin for $30 \mathrm{~min}$, the cells were incubated with primary antibody ( $\beta$ tubulin [1:100], Servicebio, Wuhan, China) for $1 \mathrm{~h}$. The nuclei were stained with DAPI (Servicebio, Wuhan, China) as a nuclear counterstain. The cellular microtubule networks were analysed by a confocal microscope. Three random fields per treatment were imaged under a Nikon Eclipse C1 microscope equipped with a Nikon DS-U3. Integrated optical density (IOD) values were recorded, and the images were analysed using Image-Pro Plus 6.0 image analysis software (Media Cybernetics, Inc., Rockville, MD, USA). ${ }^{22}$

\subsection{Docking method}

The X-ray crystal structures of tubulin bound with plinabulin (PDB ID: 5C8Y) ${ }^{23}$ and an in-house prepared co-crystal complex of tubulin with compound b (PDB ID: 5YL4) were used as the starting structures. The three-dimensional structures of the small molecules were generated in Molecular Operating Environment System (MOE) 2016.10 (Chemical Computing Group, Montreal, Canada). ${ }^{24}$ A subsequent energy minimization was carried out using Amber10:EHT force field and R-field solvation. The protein structures were prepared using QuickPrep module of MOE, and the energies were minimized through General method at $0.1 \mathrm{kcal} \mathrm{mol}^{-1} \AA^{-2} \mathrm{RMS}$ Gradient. Initial placement poses were performed with Alpha Triangle placement method. The docking poses were scored using London $\Delta G$ scoring function with five parameters, such as rotational and translational entropy, ligand flexibility, hydrogen bonding, metal ligations, and desolvation energy. At least 20 poses for each compound were retained and ranked via GBVI/WSA $\Delta G$ scoring function. The reasonable binding model of each compound was selected for further molecular dynamics simulation study.

\subsection{Molecular dynamics simulation}

Molecular dynamics (MD) simulation was performed using GROMACS (version 5.1.4) package and gromos $54 \mathrm{a} 7$ force field. $^{25,26}$ Topologies of the proteins were prepared by the pdb2gmx module of GROMACS. The ligand topology files were generated using Automated Topology Builder. ${ }^{27}$ All heterodimer systems were defined in a cubic box at least $1.0 \mathrm{~nm}$ from edge, and solvated with SPC water model. ${ }^{28}$ To neutralize the system, $\mathrm{NaCl}$ in concentration of $0.1 \mathrm{M}$ was used to replace solvent. Energy of the model proteins was minimized using the steepest descent algorithm and was converged to $F_{\max }$ (maximum force) $<10.0 \mathrm{~kJ} \mathrm{~mol}^{-1}$. Then, a $100 \mathrm{ps}$ NVT equilibration was carried out under a leap-frog integrator with a $1.4 \mathrm{~nm}$ cut-off for van der Waal (vdW) interaction, electrostatic and Linear Constraint Solver (LINCS). ${ }^{29}$ The temperature and pressure systems were regulated by Berendsen temperature ${ }^{30}$ and pressure coupling methods. ${ }^{31}$ After the system was equilibrated by a 100 ps NPT at $300 \mathrm{~K}$, MD simulation with $20 \mathrm{~ns}$ was performed with trajectories generated in time step of $2 \mathrm{fs}$, and the frames were saved every two ps. The convergence of simulation was analysed with several methods, such as root mean square deviation (RMSD), root mean square fluctuation (RMSF), radius of gyration, and number of hydrogen bonds. All coordinate frames from trajectories were superimposed on the initial conformation to remove overall translational and rotational effects. Finally, the MMPBSA binding free energy was calculated. The images were created with Pymol 0.99. ${ }^{32}$

\subsection{Free energy calculation}

The free energy calculation was performed for 250 snapshots extracted from last $2 \mathrm{~ns}$ stable MD trajectory using g_mmpbsa, ${ }^{33}$ which contained all required subroutines from GROMACS and APBS packages. The binding free energy of the protein with ligand in solvent was expressed as follows:

$$
\Delta G_{\text {bind }}=G_{\text {complex }}-\left(G_{\text {protein }}+G_{\text {ligand }}\right)
$$

$G_{\text {complex }}$ was total free energy of the protein-ligand complex. $G_{\text {protein }}$ and $G_{\text {ligand }}$ were total energy of the separated protein and ligand in solvent, respectively. Free energy of each individual $G_{\text {complex }}, G_{\text {protein }}$ and $G_{\text {ligand }}$, was estimated as follows:

$$
G_{\text {complex }}=<E_{\mathrm{MM}}>+<G_{\text {solvation }}>
$$

$E_{\mathrm{MM}}$ is the representation of the average molecular mechanics potential energy in vacuum, and $G_{\text {solvation }}$ is free energy of solvation. Molecular mechanics potential energy was calculated in vacuum using the following equation:

$$
E_{\mathrm{MM}}=E_{\text {bonded }}+E_{\text {nonbonded }}=E_{\text {bonded }}+\left(E_{\mathrm{vdW}}+E_{\text {elec }}\right)
$$

$E_{\text {bonded }}$ represents bonding interactions consisting of bond, angle, dihedral and improper interactions. $E_{\text {nonbonded }}$ interactions involved electrostatic $\left(E_{\text {elec }}\right)$ and van der Waals $\left(E_{\mathrm{vdW}}\right)$ interactions, which were modelled using Coulomb and Lennard-Jones (LJ) potential functions, respectively. Free energy $\left(G_{\text {solvation }}\right)$ of solvation was estimated as the sum of electrostatic solvation free energy $\left(G_{\text {polar }}\right)$ and apolar solvation free energy ( $\left.G_{\text {nonpolar }}\right)$ : 


$$
G_{\text {solvation }}=G_{\text {polar }}+G_{\text {nonpolar }}
$$

$G_{\text {polar }}$ and $G_{\text {nonpolar }}$ are the electrostatic and nonelectrostatic contributions to the solvation free energy, respectively. $G_{\text {polar }}$ was estimated by solving Poisson-Boltzmann (PB) equation and solvent accessible surface area (SASA). The final $\Delta G_{\text {bind }}$ value was the average value from last $2 \mathrm{~ns}$ of the MD simulation.

\section{Results and discussion}

\subsection{Inhibitory activity assay in vitro}

The anti-proliferative activities of these compounds were evaluated against BxPC-3 and NCI-H460 cell lines by SRB assay. The values of half maximal inhibitory concentration $\left(\mathrm{IC}_{50}\right)$ were calculated and are summarized in Table 1.

The $\mathrm{IC}_{50}$ values of plinabulin were $4.4 \mathrm{nM}$ and $26.2 \mathrm{nM}$ against BxPC-3 cells and NCI-H460 cells, respectively. In comparison with plinabulin, compound b had better antiproliferative activities; its $\mathrm{IC}_{50}$ values were $0.9 \mathrm{nM}$ against BxPC-3 cell lines and $4.1 \mathrm{nM}$ against NCI-H460 cell lines. These results indicated that the benzoyl group substituted on A-ring could enhance the bioactivity of compound $\mathbf{b}$. As shown in Table 1, compound $\mathbf{c}$, with a para-F atom at the phenyl group, displayed the highest biological activity, and its $\mathrm{IC}_{50}$ values were $0.7 \mathrm{nM}$ and $3.8 \mathrm{nM}$ against BxPC-3 and NCI-H460, respectively.

Table 1 In vitro anti-proliferative activity of DKP derivatives in BxPC-3 and $\mathrm{NCl}-\mathrm{H} 460$ cell lines, and their inhibition rate of tubulin polymerization at $5 \mu \mathrm{M}^{a}$

\begin{tabular}{|c|c|c|c|}
\hline & $\begin{array}{l}\mathrm{IC}_{50} \text { BxPC-3 } \\
(\mathrm{nM})\end{array}$ & $\begin{array}{l}\mathrm{IC}_{50} \text { NCI-460 } \\
(\mathrm{nM})\end{array}$ & $\begin{array}{l}\text { Inhibition rate } \\
\text { of tubulin } \\
\text { polymerization }(5 \mu \mathrm{M})\end{array}$ \\
\hline Plinabulin & $4.4 \pm 1.10$ & $26.2 \pm 3.20$ & $68.9 \%$ \\
\hline a & $306.4 \pm 41.40$ & $>1000$ & $12.8 \%$ \\
\hline b & $0.9 \pm 0.04$ & $4.1 \pm 0.60$ & $72.2 \%$ \\
\hline c & $0.7 \pm 0.03$ & $3.8 \pm 1.20$ & $92.5 \%$ \\
\hline d & $56.8 \pm 4.80$ & $51.7 \pm 6.60$ & $66.1 \%$ \\
\hline e & $5.0 \pm 0.90$ & $27.2 \pm 3.10$ & $61.1 \%$ \\
\hline f & $91.7 \pm 6.00$ & $388.7 \pm 53.80$ & $9.9 \%$ \\
\hline
\end{tabular}

${ }^{a}$ All these values were indicated as the mean \pm SD of three independent experiments.
In contrast, plinabulin had better anti-proliferative activity than that of compound a $\left(\mathrm{IC}_{50}=306 \mathrm{nM}\right.$ for BxPC-3 cells and $\mathrm{IC}_{50}>1000 \mathrm{nM}$ for NCI-H460 cells). Similarly, compound $\mathbf{b}$ had better anti-proliferative activity than that of compound $\mathbf{d}\left(\mathrm{IC}_{50}=\right.$ $56.8 \mathrm{nM}$ for BxPC-3 cell lines, $\mathrm{IC}_{50}=51.7 \mathrm{nM}$ for NCI-H460 cells). These results showed that the replacement of tert-butyl group with methyl group at C-ring could decrease the bioactivity. Since the substituted group at C-ring could also influence the bioactivity, we further explored compound $\mathbf{e}$ with a pyridine group at C-ring, which had comparable bioactivity with compound $\mathbf{b}\left(\mathrm{IC}_{50}=5.0 \mathrm{nM}\right.$ for BxPC-3, $\mathrm{IC}_{50}=27.2 \mathrm{nM}$ for NCIH460). However, in contrast, compound $\mathbf{f}\left(\mathrm{IC}_{50}=91.7 \mathrm{nM}\right.$ for BxPC-3, IC $_{50}=388.7 \mathrm{nM}$ for NCI-H460) had a benzyl-substituted group at C-ring, and showed greatly reduced bioactivity compared to compound $\mathbf{b}$. These results revealed that the intramolecular hydrogen bond was essential to maintain bioactivity.

To demonstrate the binding activity, fluorescence-based tubulin polymerization assay was measured with time (Fig. 3a). Inhibition rates of plinabulin and compounds a-f were found to be $68.9 \%, 12.8 \%, 72.2 \%, 92.5 \%, 66.1 \%, 61.1 \%$ and $9.9 \%$, respectively (Table 1 ). In contrast, the polymerization inhibition activities were consistent with the $\mathrm{IC}_{50}$ values against BxPC-3 and NCI-H460 cell lines as shown in Fig. 3b.

An immunofluorescence assay was performed to confirm whether compounds a-f could disrupt the microtubule dynamics in cells. As shown in Fig. 4a, the microtubule network in BxPC-3 cells was well-defined and wrapped around the uncondensed cell nucleus; in contrast, the formation of spindles in cells after exposure to the compounds demonstrated distinct abnormalities. Furthermore, semi-quantitative analyses of these compounds exhibited the disruption of tubulin polymerizations as shown in Fig. $4 \mathrm{~b}$, which could be considered as direct evidence. Again, the inhibition activities were also consistent with the anti-proliferative activities as shown in Fig. 3b.

\subsection{Docking results}

Molecular docking simulation of plinabulin and compounds af were performed using the dock module of MOE software package. The reasonable binding poses in colchicine binding pocket are displayed in Fig. 5. The docking results indicated

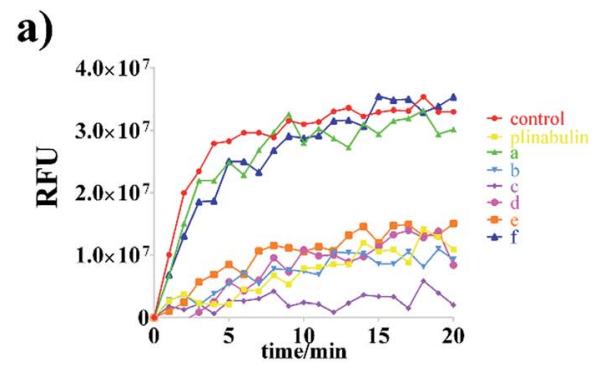

b)

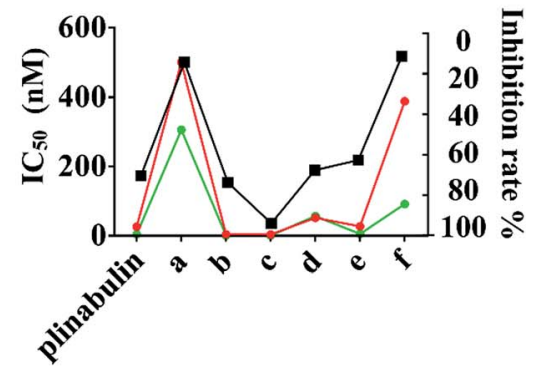

Fig. 3 Effects of plinabulin and compounds a-f at $5 \mu \mathrm{M}$ on the tubulin polymerization. (a) Tubulin polymerization inhibition activities measured with time at $37^{\circ} \mathrm{C}$. (b) Comparison of $\mathrm{IC}_{50}$ values (BxPC-3: green; $\mathrm{NCl}-\mathrm{H} 460$ : red) and tubulin inhibition rate (black) of DKP derivatives. 
a)

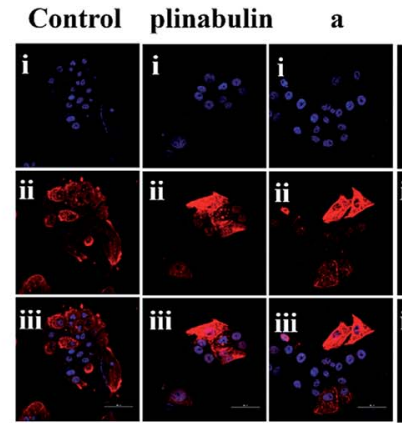

b)

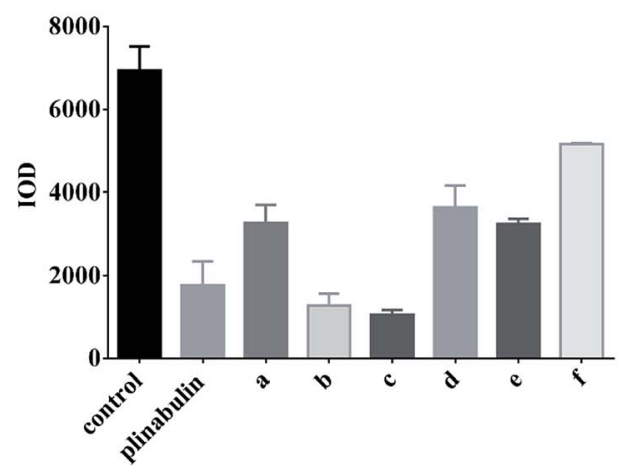

Fig. 4 Immunofluorescence assay of plinabulin and compounds a-f inhibition of tubulin polymerization in vitro. (a) Confocal images of DKP derivatives (5 nM) disrupting the mitotic spindles in BxPC-3 cell. (i) Nuclear (blue); (ii) tubulin (red); (iii) (i) and (ii) were overlapped. Scale bar is $50 \mu \mathrm{m}$. (b) Semi-quantitative analysis of the inhibition of tubulin polymerization.
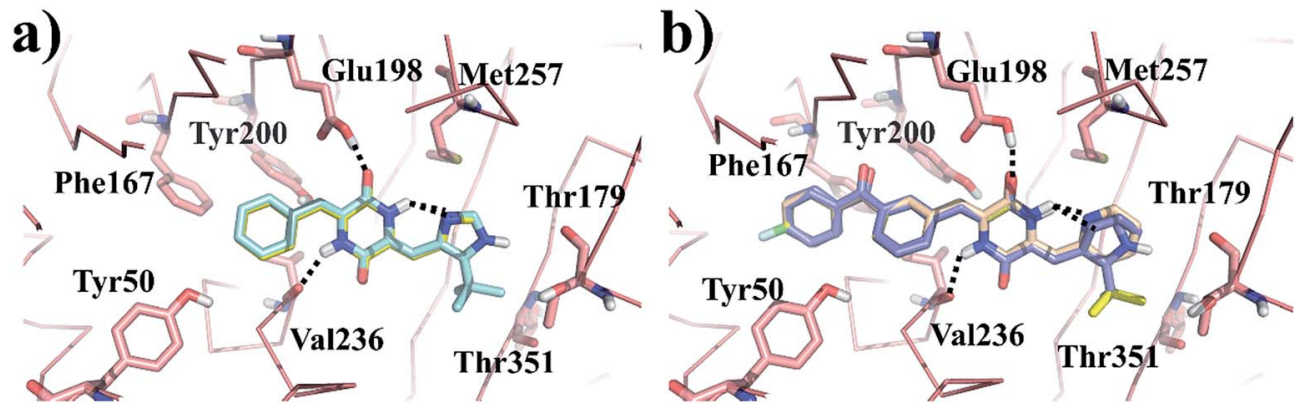

Fig. 5 The reasonable binding poses of the inhibitors in tubulin. (a) Plinabulin and compound a in the pocket $5 \mathrm{C} 8 \mathrm{Y}$. (b) Compounds $b-f$ in the pocket $5 Y L 4$.

that all derivatives formed a crucial hydrogen bonding interaction between the diketopiperazine backbone and Val236 of $\beta$ tubulin. For MD simulation, considering the flexibility of tubulin, the pocket of 5C8Y was selected for the binding poses of plinabulin and compound a, while the pocket of 5YL4 was selected for the binding poses of compounds $\mathbf{b}-\mathbf{f}$.

\subsection{Structural stability}

It was crucial to obtain a stable molecular dynamics trajectory for subsequent analysis. The time dependent root mean square deviation (RMSD) of the backbone was calculated to evaluate the structural stability. As shown in Fig. 6a, all systems reached stability after $22 \mathrm{~ns}$. The residues-dependent root mean squared fluctuation (RMSF) of the side chain was calculated to assess the flexibility of residues. In general, the RMSF plots presented low fluctuations in $\alpha$-helix and $\beta$-sheet, and high peaks in the loops. Moreover, H1-B2 loop (residues 35-60) and M-loop (residues 272-290) were involved in contacting the protofilaments ${ }^{34}$ and had higher structural flexibility (Fig. 6b).

\subsection{Structural analysis}

To reveal the effects of DKP derivatives on the overall structure of tubulin, the structures of tubulin-apo and tubulin-plinabulin were superimposed before and after molecular dynamics simulation (Fig. 7a or b). In comparison with tubulin-apo, the conformation of tubulin-plinabulin clearly changed. Furthermore, the gyration radii of tubulin-apo and tubulin-plinabulin were 2.93 and $3.02 \mathrm{~nm}$, respectively (Fig. 7c). The possible 
a)

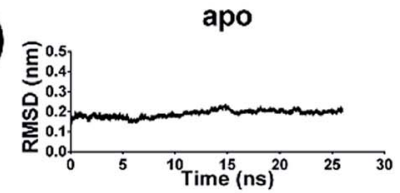

Compound $\mathrm{c}$

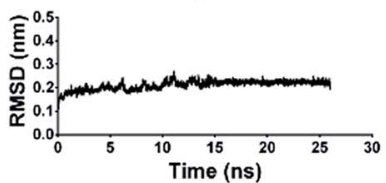

b)

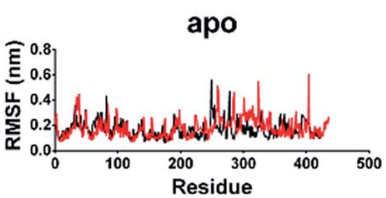

compound $\mathrm{c}$

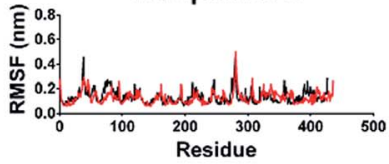

plinabulin

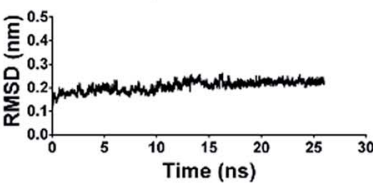

Compound d

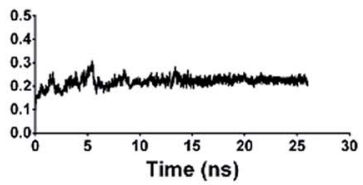

plinabulin

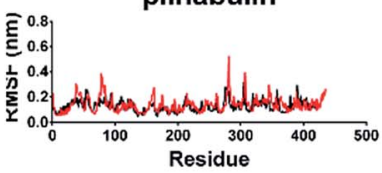

compound d

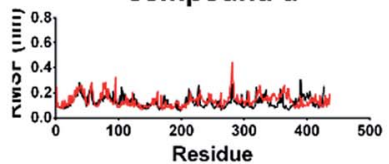

Compound a

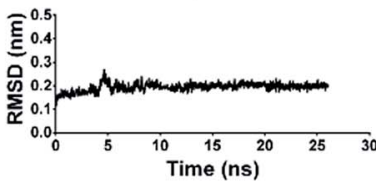

Compound e

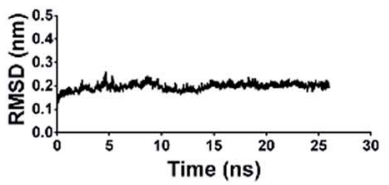

compound a

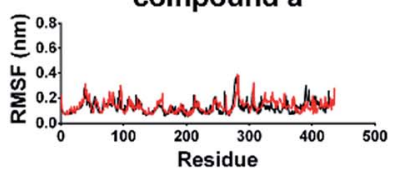

compound e

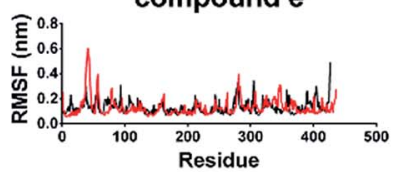

Compound $\mathrm{b}$

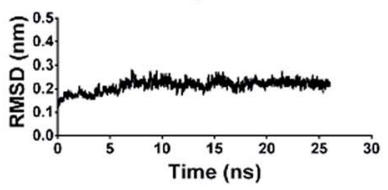

Compound $\mathbf{f}$

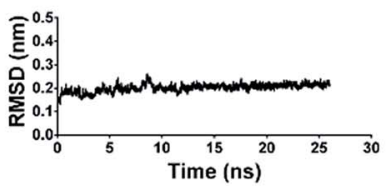

compound b

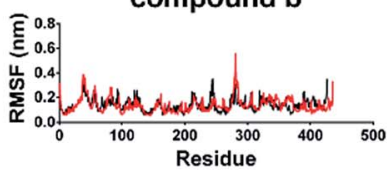

compound $f$

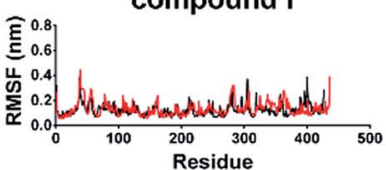

Fig. 6 Structural stability of the tubulin-apo and tubulin-inhibitors complexes. (a) Backbone RMSD of dipolymer (black). (b) RMSF of side chain in $\alpha$-tubulin (red) and $\beta$-tubulin (black).

a)

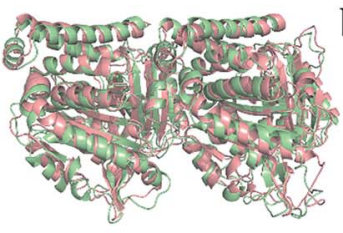

d)

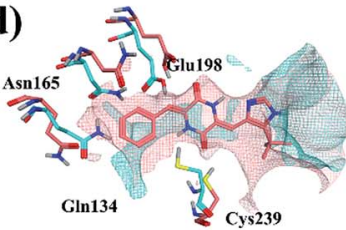

b)

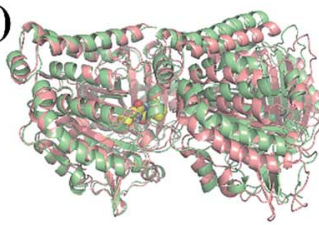

e)

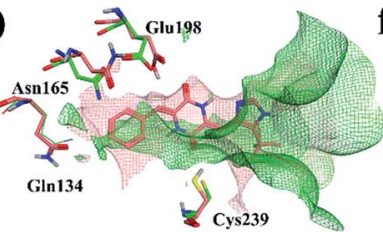

c)

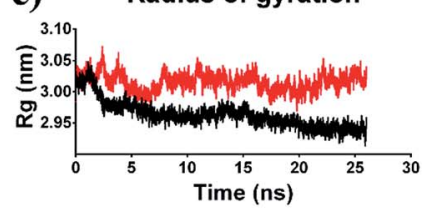

f)

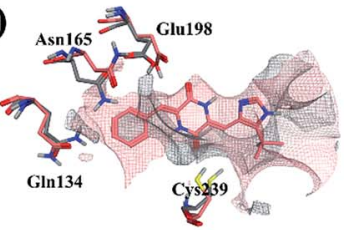

Fig. 7 Structural analysis of MD simulation. Overview of the structures of (a) tubulin-apo at 0 ns (pink) and 26 ns (light green); (b) tubulinplinabulin at 0 ns (pink) and 26 ns (light green); (c) radius of gyration of tubulin-plinabulin (red) and tubulin-apo (black) with time; (d) the pocket comparison of tubulin-apo of 0 ns (pink) and 26 ns (cyan); (e) the pocket comparison of the crystal structures of tubulin bound with plinabulin (pink) (PDB ID: 5C8Y) and with colchicine (green) (PDB ID: 1SA0); (f) the pocket comparison of 5C8Y (pink) and tubulin-apo (grey) (PDB ID: 3HKB).

reason was that the DKP derivatives could inhibit the microtubules' polymerization through altering the conformation of tubulin. As shown in Fig. 7d, the pink surface and the cyan surface represent the plinabulin binding pocket before and after MD simulation, respectively. The binding pocket of plinabulin in tubulin-apo faded away after $26 \mathrm{~ns}$ of MD simulation. In addition, the crystal structures of tubulin with colchicine (PDB ID: 1SA0 (ref. 8)) and plinabulin (PDB ID: 5C8Y ${ }^{23}$ ) were superposed with $\beta$-tubulin (Fig. 7e). This indicated that plinabulin was slightly overlapped with colchicine in the binding site and the active site pockets showed that plinabulin could not effectively fit into the colchicine pocket. Therefore, we assumed that the binding pocket of plinabulin was induced and did not exist in original tubulin polymers. In order to verify this, the structures of tubulin without any ligand (PDB ID: $3 \mathrm{HKB}^{35}$ ) and tubulin-plinabulin were superimposed with $\beta$-tubulin. As shown in Fig. 7f, no binding pockets were observed for plinabulin in 3HKB or in the simulated tubulin-apo structure after 26 ns, which conformed to our assumption.

Based on the ligand-position RMSD results, plinabulin with $t$-butyl moiety was more stable than compound a with methyl moiety during MD simulation (Fig. 8a). A migration of compound a was observed when the structure of tubulina complex was superimposed onto the structure of tubulin- 
a)
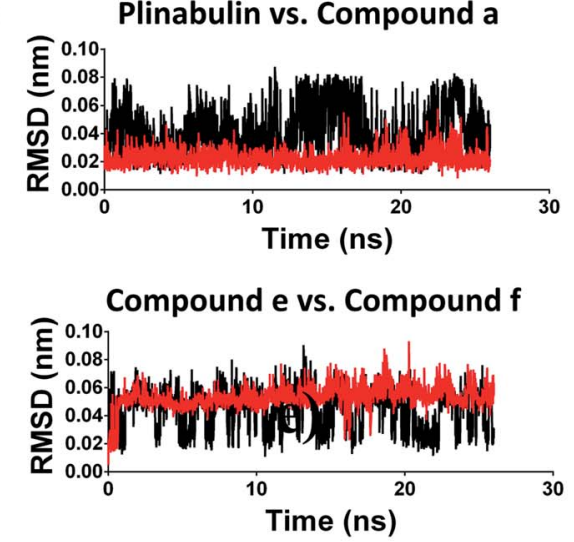

c)

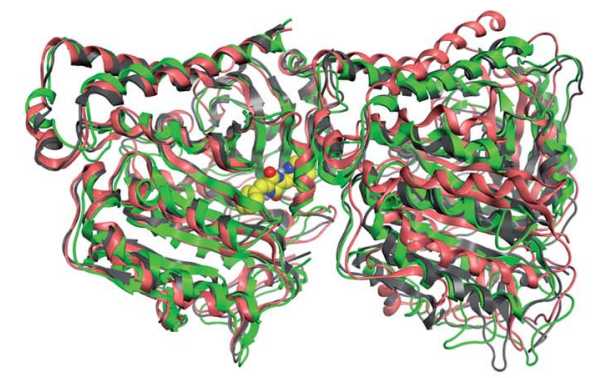

Compound e

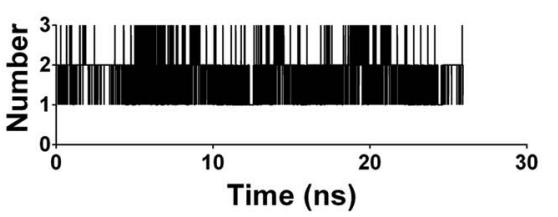

b)

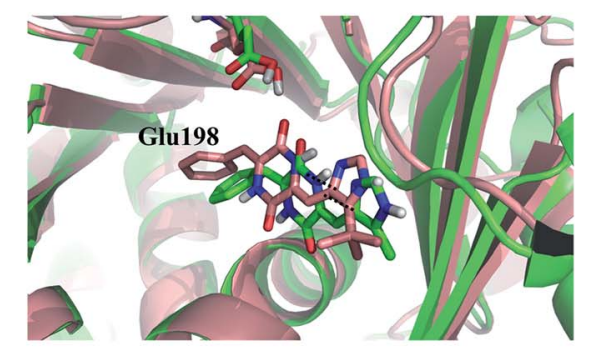

d)

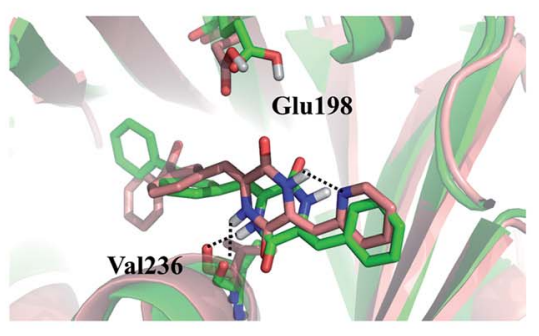

Compound $f$

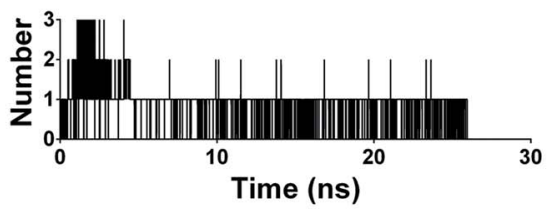

e)

Fig. 8 (a) Ligand-position RMSDs of plinabulin, compound a, compound e, and compound f; (b) structure analysis of MD simulation: tubulinplinabulin (pink) vs. tubulin-a (green) (c) overview of the structures of tubulin-e (pink) and tubulin-f (green) after MD simulation vs. tubulin- $f$ (grey) before MD simulation; (d) structure analysis of MD simulation: tubulin-e (pink) vs. tubulin-f (green); (e) number of hydrogen bonds: compound e vs. compound f.

plinabulin complex (Fig. 8b). The structural analysis indicated that compound a shifted outward and the methyl group shifted toward the corresponding $t$-butyl group location. We assumed that the $t$-butyl moiety could interact with tubulin in a hydrophobic manner, which was the major contribution to the inhibitor's binding.

Compound e with an intramolecular hydrogen bond was stable in the pocket compared with compound $\mathbf{f}$, which lacked the intramolecular hydrogen bond (Fig. 8a). The structure of tubulin-f complex had a deflection in B-ring and C-ring compared with the tubulin-e complex (Fig. 8d). This deflection made compound f lose the hydrogen bond with Glu198; this result was also proved by analysis of the number of hydrogen bonds (Fig. 8e). The overall structural superimposition indicated that the tubulin-f complex was stable before and after MD simulation, while the tubulin-e complex was bent greatly after MD simulation (Fig. 8c), a conformation change which could hinder the assembly of tubulin. ${ }^{36}$ These results were consistent with the biological assay that compound e had better anti-proliferative and inhibition activities of microtubules in comparison with compound $\mathbf{f}$. Therefore, we assumed that the intramolecular hydrogen bond of this ligand was important for forming a reasonable binding mode and further inhibiting tubulin polymerization.

\subsection{Binding free energy calculation}

Combined with MD simulation, the binding free-energy calculation method has become a powerful tool for quantitative determination of the protein-ligand interaction. In total, 250 snapshots extracted from last 2 ns of MD trajectories were used to analyse the binding free energy with MM-PBSA method. As shown in Table 2 , the binding free energy $\left(\Delta G_{\text {bind }}\right)$ of plinabulin and compounds a-f were $-100.82,-83.96,-171.32,-193.86$, $-92.47,-148.09$, and $-146.15 \mathrm{~kJ} \mathrm{~mol}^{-1}$, respectively.

According to energy components of the binding free energy, the major favourable contributor of the inhibitor binding was van der Waals interaction $\left(\Delta E_{\mathrm{vdw}}\right)$. Moreover, the electrostatic energy 
Table 2 The energetic terms obtained from MM-PBSA calculation for DKP derivatives binding to tubulin $\left(\mathrm{kJ} \mathrm{mol}^{-1}\right)$

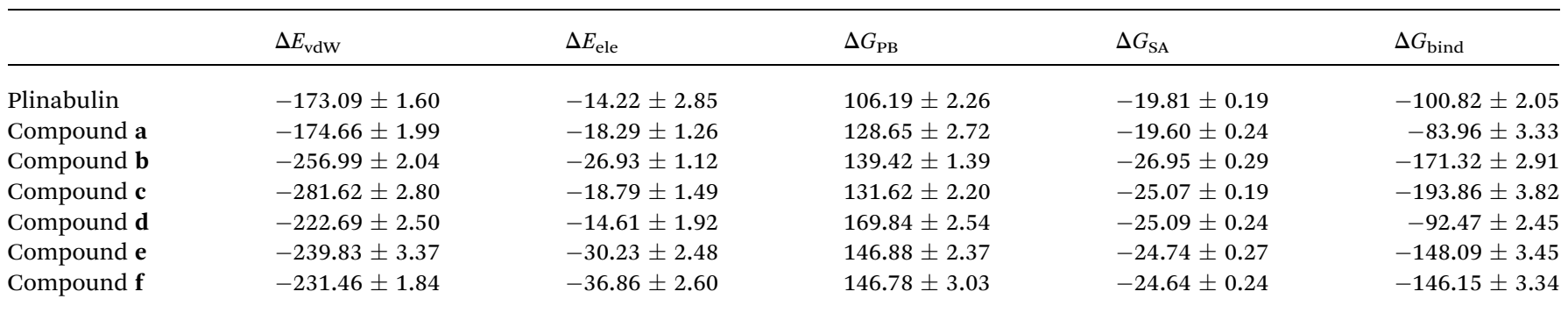

$\left(\Delta E_{\text {ele }}\right)$ and nonpolar solvation free energy $\left(\Delta G_{\mathrm{SA}}\right)$ were also favourable for the binding. In addition, the non-polar interactions $\left(\Delta E_{\mathrm{vdW}}+\Delta G_{\mathrm{SA}}\right)$ were the dominating force for inhibitor binding. These results suggested that optimization of van der Waals interactions between inhibitors and tubulin might improve their biological activities. Therefore, we concluded that hydrophobic interaction played an important role in the development of potent tubulin polymerization inhibitors.

To identify the key residues for these inhibitors binding to tubulin, the binding free energies were decomposed into individual residues. $\Delta G_{\text {bind }}$ energy contributions from each residue are represented in Fig. $\mathrm{S} 1 . \dagger$ The key residues are summarized in Fig. 9. The results revealed that the attractive contributions primarily originate from Glu183 of $\alpha$-tubulin and $\beta$-tubulin residues, such as Asn165, Tyr200, Asp249, Leu253, Met257, Ala314, Ile316, and Ile368. Docking results indicated that DKP ring could form a hydrogen bond with the protonated carboxyl group of Glu198 of $\beta$-tubulin. However, the energy decomposition indicated that DKP ring had unfavourable interaction with Glu198 of $\beta$-tubulin, which probably resulted a)

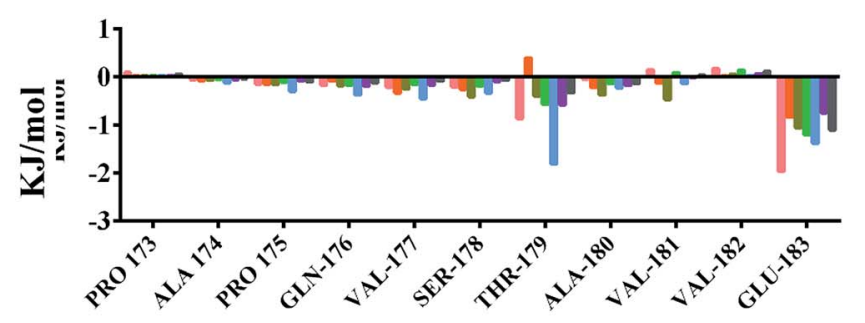

b)
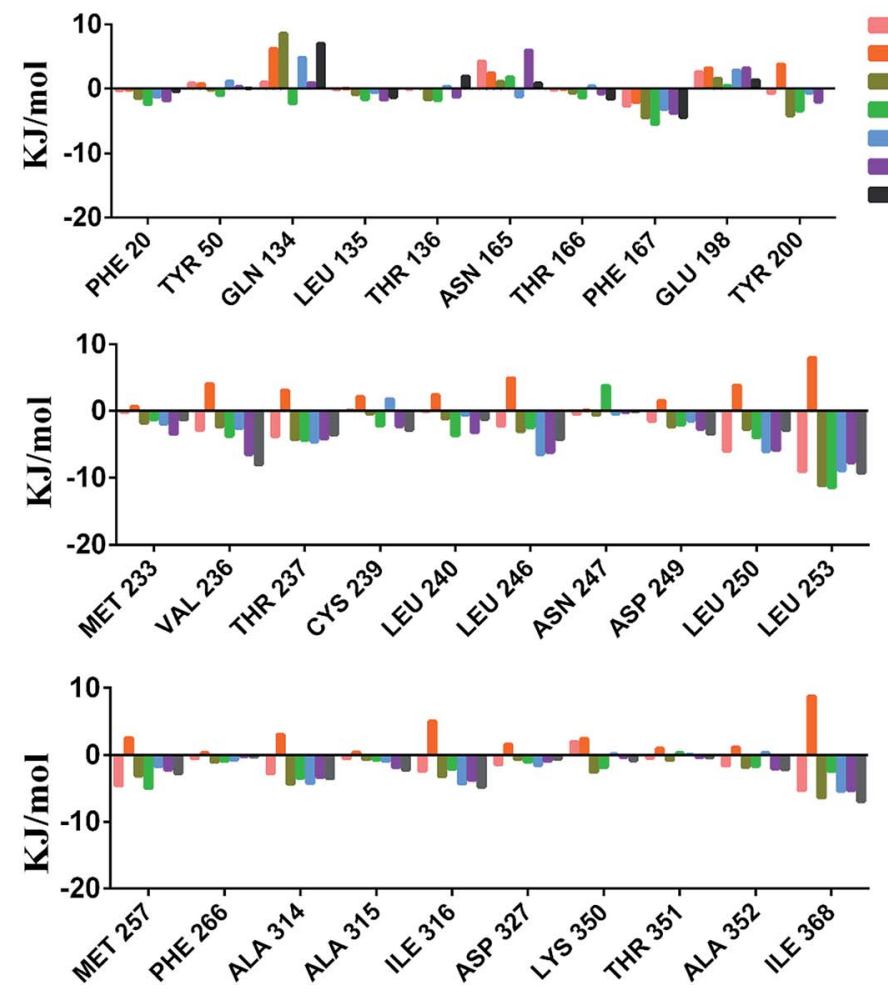

Fig. 9 Contributions of the key residues of plinabulin and compounds a-f. (a) $\alpha$-tubulin. (b) $\beta$-tubulin. 
from the electron repulsion between the other carboxylic oxygen of Glu198 and DKP ring (Fig. S2†). The potency could be improved by removing this unfavourable interaction.

\section{Conclusion}

In this study, both molecular docking and MD simulation were used to explore the interaction between tubulin and DKP derivatives. The binding free energy decomposition showed that van der Waals interactions were dominant for the binding affinity. Further, the total binding free energy was decomposed into each residue. The binding free energy calculation revealed that the binding affinity between ligand and tubulin was mostly contributed from $\beta$-tubulin. The results also indicated that the key residues for tubulin binding were Asn165, Tyr200, Val236, Asp249, Leu253, Met257, Ala314, Ile316, and Ile368, which created a hydrophobic pocket at $\beta$-tubulin. In addition, the trend of the binding free energy of these inhibitors was in agreement with the trend of their biological activities (Table 1).

In summary, this investigation provided a molecular level understanding of the mechanism, and provided valuable information for further rational anticancer drug design.

\section{Conflicts of interest}

There are no conflicts to declare.

\section{Abbreviations}

$\begin{array}{ll}\text { MD } & \text { Molecular Dynamics } \\ \text { MM- } & \text { Molecular Mechanics/Poisson-Boltzmann Surface } \\ \text { PBSA } & \text { Area } \\ \text { vdW } & \text { van der Waal } \\ \text { CBSI } & \text { Colchicine Binding Site Inhibitor } \\ \text { MTs } & \text { Microtubules } \\ \text { MTAs } & \text { Microtubule Targeting Agents } \\ \text { FDA } & \text { Food and Drug Administration } \\ \text { MDR } & \text { Multidrug Resistance } \\ \text { IOD } & \text { Integrated Optic Density } \\ \text { PDB } & \text { Protein Data Bank } \\ \text { SRB } & \text { Sulforhodamine B } \\ \text { MS } & \text { Mass Spectrometry } \\ \text { NMR } & \text { Nuclear Magnetic Resonance } \\ \text { HPLC } & \text { High Performance Liquid Chromatography }\end{array}$

\section{Acknowledgements}

We acknowledge the supports of "Zhufeng Scholar Program" of Ocean University of China (841412016), "Outstanding Talents Plan” of Qingdao (15-10-3-15-(34)-zch), and Aoshan Talents Cultivation Program of Qingdao National Laboratory for Marine Science and Technology (No. 2017ASTCP-OS08) to Dr Wenbao $\mathrm{Li}$, and the Youth Special Fund of Qingdao (16-5-1-61-jch) to Dr Yanyan Chu. We also acknowledge that some compounds in this paper were provided by our colleagues, Mr Zhongpeng Ding, Dr Yingwei Hou, Mr Zhangyu Fu and Mr Mingxu Ma.

\section{References}

1 J. C. Zabala and N. J. Cowan, Cell Motil. Cytoskeleton, 1992, 23, 222-230.

2 C. H. S. Aylett, J. Lowe and L. A. Amos, Int. Rev. Cell Mol. Biol., 2011, 292, 1-71.

3 K. H. Downing and E. Nogales, Curr. Opin. Struct. Biol., 1998, 8, 785-791.

4 J. Howard and A. A. Hyman, Nature, 2003, 422, 753-758.

5 D. Fanale, G. Bronte, F. Passiglia, V. Calo, M. Castiglia, F. Di Piazza, N. Barraco, A. Cangemi, M. Teresa Catarella, L. Insalaco, A. Listi, R. Maragliano, D. Massihnia, A. Perez, F. Toia, G. Cicero and V. Bazan, Anal. Cell. Pathol., 2015, 2015(15), 690916.

6 J. Lowe, H. Li, K. H. Downing and E. Nogales, J. Mol. Biol., 2001, 313, 1045-1057.

7 B. Gigant, C. Wang, R. B. G. Ravelli, F. Roussi, M. O. Steinmetz, P. A. Curmi, A. Sobel and M. Knossow, Nature, 2005, 435, 519-522.

8 R. B. G. Ravelli, B. Gigant, P. A. Curmi, I. Jourdain, S. Lachkar, A. Sobel and M. Knossow, Nature, 2004, 428, 198-202.

9 A. E. Prota, K. Bargsten, P. T. Northcote, M. Marsh, K.-H. Altmann, J. H. Miller, J. F. Diaz and M. O. Steinmetz, Angew. Chem., Int. Ed., 2014, 53, 1710.

10 P. Seve and C. Dumontet, Lancet Oncol., 2008, 9, 168-175.

11 M. A. Jordan and L. Wilson, Nat. Rev. Cancer, 2004, 4, 253-265.

12 Y. Finkelstein, S. E. Aks, J. R. Hutson, D. N. Juurlink, P. Nguyen, G. Dubnov-Raz, U. Pollak, G. Koren and Y. Bentur, Clin. Toxicol., 2010, 48, 407-414.

13 K. Ohsumi, R. Nakagawa, Y. Fukuda, T. Hatanaka, Y. Morinaga, Y. Nihei, K. Ohishi, Y. Suga, Y. Akiyama and T. Tsuji, J. Med. Chem., 1998, 41, 3022-3032.

14 K. Kanoh, S. Kohno, T. Asari, T. Harada, J. Katada, M. Muramatsu, H. Kawashima, H. Sekiya and I. Uno, Bioorg. Med. Chem. Lett., 1997, 7, 2847-2852.

15 K. Kanoh, S. Kohno, J. Katada, J. Takahashi and I. Uno, J. Antibiot., 1999, 52, 134-141.

16 https://clinicaltrials.gov/show/NCT02504489.

17 Y. Yamazaki, K. Tanaka, B. Nicholson, G. Deyanat-Yazdi,

B. Potts, T. Yoshida, A. Oda, T. Kitagawa, S. Orikasa,

Y. Kiso, H. Yasui, M. Akamatsu, T. Chinen, T. Usui,

Y. Shinozaki, F. Yakushiji, B. R. Miller, S. Neuteboom,

M. Palladino, K. Kanoh, G. K. Lloyd and Y. Hayashi, J. Med. Chem., 2012, 55, 1056-1071.

18 Y. Hayashi, H. Takeno, T. Chinen, K. Muguruma, K. Okuyama, A. Taguchi, K. Takayama, F. Yakushiji, M. Miura, T. Usui and Y. Hayashi, ACS Med. Chem. Lett., 2014, 5, 1094-1098.

19 Z. Ding, Y. Hou, S. Wang, T. Sun, M. Ma, H. Guan and W. Li, Mol. Diversity, 2017, 21, 577-583.

20 D. Bonne, C. Heusele, C. Simon and D. Pantaloni, J. Biol. Chem., 1985, 260, 2819-2825.

21 A. V. Singh, M. Bandi, N. Raje, P. Richardson, M. A. Palladino, D. Chauhan and K. C. Anderson, Blood, 2011, 117, 5692-5700. 
22 P. Singh, A. Anand and V. Kumar, Eur. J. Med. Chem., 2014, 85, 758-777.

23 Y. Wang, H. Zhang, B. Gigant, Y. Yu, Y. Wu, X. Chen, Q. Lai, Z. Yang, Q. Chen and J. Yang, FEBS J., 2016, 283, 102-111.

24 D. Pogoryelov, A. Krah, J. D. Langer, O. Yildiz, J. D. FaraldoGomez and T. Meier, Nat. Chem. Biol., 2010, 6, 891-899.

25 M. Christen, P. H. Hunenberger, D. Bakowies, R. Baron, R. Burgi, D. P. Geerke, T. N. Heinz, M. A. Kastenholz, V. Krautler, C. Oostenbrink, C. Peter, D. Trzesniak and W. F. Van Gunsteren, J. Comput. Chem., 2005, 26, 1719-1751. 26 N. Schmid, A. P. Eichenberger, A. Choutko, S. Riniker, M. Winger, A. E. Mark and W. F. Gunsteren, Eur. Biophys. J., 2011, 40, 843-856.

27 K. B. Koziara, M. Stroet, A. K. Malde and A. E. Mark, J. Comput.-Aided Mol. Des., 2014, 28, 221-233.

28 S. Miyamoto and P. A. Kollman, J. Comput. Chem., 1992, 13, 952-962.
29 S. Amiri, M. S. P. Sansom and P. C. Biggin, Protein Eng., Des. Sel., 2007, 20, 353-359.

30 H. J. C. Berendsen, J. R. Grigera and T. P. Straatsma, J. Phys. Chem., 1987, 91, 6269-6271.

31 M. Parrinello and A. Rahman, J. Appl. Phys., 1981, 52, 71827190.

32 J. Curuksu, J. Sponer and M. Zacharias, Biophys. J., 2009, 97, 2004-2013.

33 R. Kumari, R. Kumar and A. Lynn, J. Chem. Inf. Model., 2014, 54, 1951-1962.

34 R. Zhang, G. M. Alushin, A. Brown and E. Nogales, Cell, 2015, 162, 849-859.

35 A. Dorleans, B. Gigant, R. B. Ravelli, P. Mailliet, V. Mikol and M. Knossow, Proc. Natl. Acad. Sci. U. S. A., 2009, 106, 1377513779.

36 L. Pecqueur, C. Duellberg, B. Dreier, Q. Jiang, C. Wang, A. Pluckthun, T. Surrey, B. Gigant and M. Knossow, Proc. Natl. Acad. Sci. U. S. A., 2012, 109, 12011-12016. 